Graham Doig $\cdot$ Zebulan Johnson $\cdot$ Rachel Mann

\title{
Interaction of shock tube exhaust flow with a non-pre-mixed flame
}

\section{Introduction}

Much of the discussion in the public domain relating to shockwave interaction with flames, and the use of high explosives to extinguish, for instance, oil and gas well fires, is anecdotal and outside of scientific literature. The result is a lack of consistent insight into the physical mechanisms involved. It has been proposed by several researchers that the shock waves and the strong vortex rings from detonations are effective in extinguishing large-scale fires (Akhmetov et al. 1980, 2001, 2009; Bliznetsov et al. 2001). To date, methodical laboratory shock tube work in controlled conditions for simple flame cases has not been reported.

Therefore, a preliminary laboratory-scale study was devised to investigate, qualitatively, the interaction between the flow exhausting from an open-ended shock tube and a standard Bunsen burner flame. The shock tube was a basic, compressed air-driven device not capable of producing exactly the kind of temporal pressure profile associated with explosive events (i.e. a Friedlander-esque waveform) (Chandra et al. 2012). However, the flow fields obtained resulted in many interactions not previously observed and documented. This was possible due to the complex flow features emanating from the tube exit, including strongly rotating vortices and a central jet of flow following the initial shock wave (Jiang et al. 2003; Kashimura et al. 2000; Onodera et al. 1998).

The flow from the shock tube was expected to be in the low-supersonic range within the immediate vicinity of the exit, with the leading shock and jet both dissipating significantly downstream with an accompanying reduction in planarity. Therefore, the effect of the exhaust flow on the Bunsen safety (yellow) non-pre-mixed flame was examined at $50 \mathrm{~mm}$ and $500 \mathrm{~mm}$ from the tube exit to investigate the range of interactions possible with this setup. Multiple stagnation pressures in the driver section (from 140 to $620 \mathrm{kPa}$ ) were used to examine the influence of Mach number at the exit (1.1-1.4). The shock tube had a $44 \mathrm{~mm} \times 47 \mathrm{~mm}$ rectangular cross-sectional exit, a 1,790 $\mathrm{mm}$ long driver section, and a 2,195 $\mathrm{mm}$ driven section. The visualization was captured at 6,000-10,000 fps using a Photron APX-RS, with an exposure time of $20 \mu \mathrm{s}$. A conventional z-type schlieren arrangement was utilized with a vertical knife-edge cutoff.

\section{Results and discussion}

Wherever the flame was placed and whatever the oncoming Mach number of the flow, the mechanism for the flame being extinguished was the same; the impulsive force of the air following the initial shockwave

G. Doig $(\bowtie) \cdot$ Z. Johnson $\cdot$ R. Mann

School of Mechanical and Manufacturing Engineering, The University of New South Wales,

Sydney, NSW 2052, Australia

E-mail: g.doig@unsw.edu.au

Tel.: +61-9-3854121 
was in all cases responsible for pushing the flame off its fuel source, leading to rapid cessation of combustion. The shock itself passed through the Bunsen region with little immediate influence other than a slight perturbation to the flame occurring at a timescale several orders of magnitude faster than natural fluctuations. When the core jet flow of the shock tube exhaust was in-line with the flame, the jet produced the extinguishing effect. When the flame was offset from the line of the jet, the vortex rings produced at the tube exit extinguished the flames rapidly with strong, turbulent rotating flow propagating downstream.

Figure 1 indicates the interactions obtained for driver stagnation pressures from 205 to $620 \mathrm{kPa}$ (corresponding to outlet flow conditions of approximately Mach $1.15-1.5 \pm 0.03$ ) when the safety flame was positioned $50 \mathrm{~mm}$ from the shock tube exit. The initial spherical shock moved too rapidly to be captured effectively in any single frame, though the vortex ring structure was observable (albeit offset from the focal plane). For the lowest Mach number of 1.15 (left), the strong jet flow from the tube exit pushes the flame off its fuel source between frames (ii) and (iii), and by frame (iv), $2 \mathrm{~ms}$ after the initial exhaust of flow from the tube enters the field of view at $t^{*}=0$, the combustion process has ceased. Only turbulent hot air remains, edging out of the field of view. At the higher Mach numbers, the process is the same; however, the interaction happens on a shorter timeframe. After the initial blast removes the flame from the gas supply with greater force, the formation of attached shockwaves on the Bunsen lip is visible and no re-ignition of the flame occurs in the supersonic expanding jet region for the duration of this established flow field (approx. $10 \mathrm{~ms}$ ).

When the flame was placed $500 \mathrm{~mm}$ from the tube exit, a more straightforward interaction resulted in the extinguishment (Fig. 2a). The initial shock, significantly weakened at this distance (potentially a subsonic pressure wave), passes through the flame between frames (i) and (ii) - the wave itself is blurred due to the exposure time of the images. The oncoming jet of subsonic air pushes the flame off the gas source leading to the rapid extinguishment previously observed. The foremost and rearward extents of the flame are compressed together, between frames (iii) and (iv) into a narrow front exhibiting a notable but slight spherical aspect due to the three-dimensionality of the flow from the tube exhaust.

When the flame was offset from the centreline of the exhaust range, a very different interaction was observed, as shown in Fig. 2b, c). Here, the strong ring vortices produced at the corners of the tube exit and
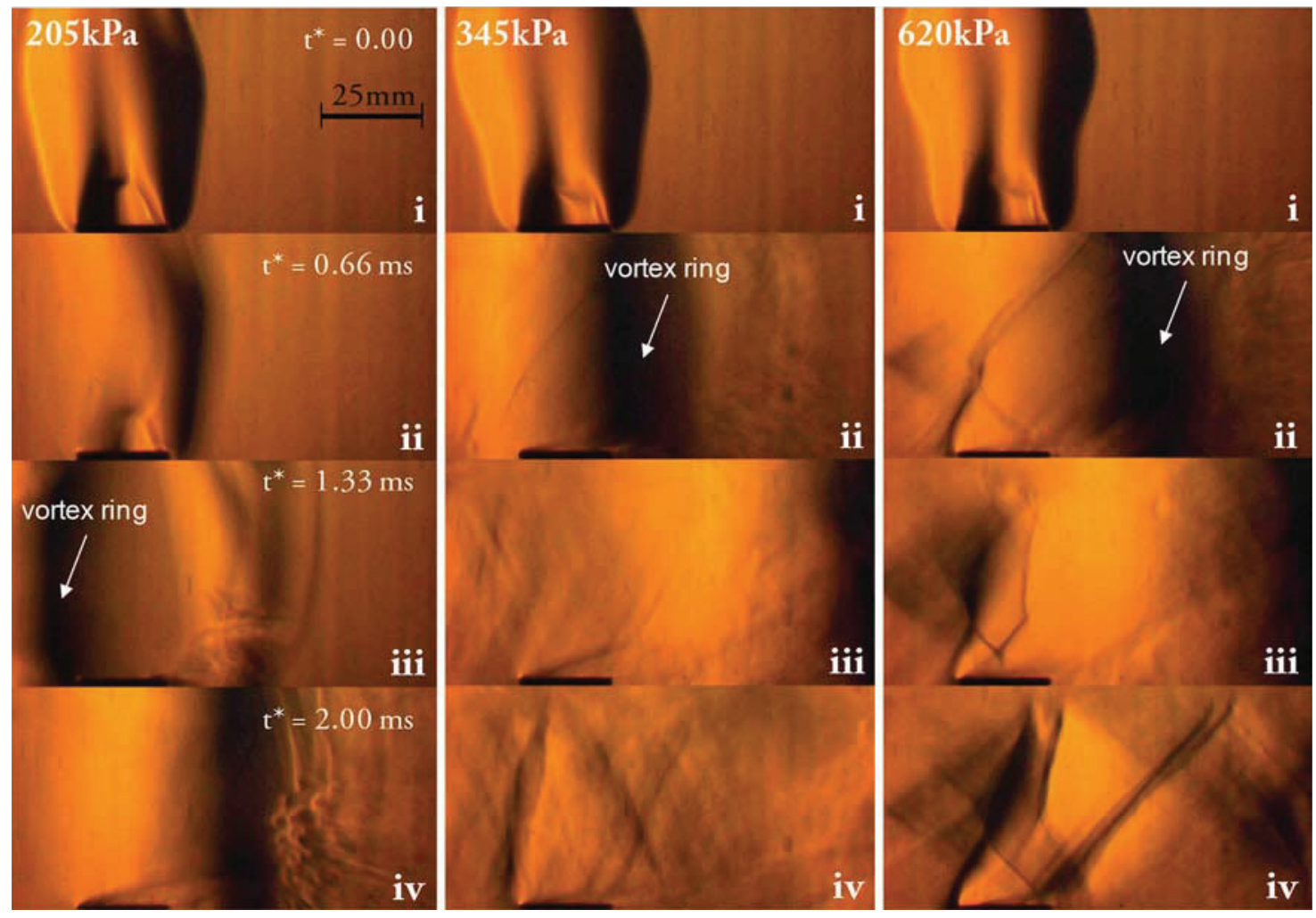

Fig. 1 Sequences from cases with flame positioned $50 \mathrm{~mm}$ from the shock tube exit, spanning a total time of approximately $2 \mathrm{~ms}$ for different driver stagnation pressures and Mach numbers 


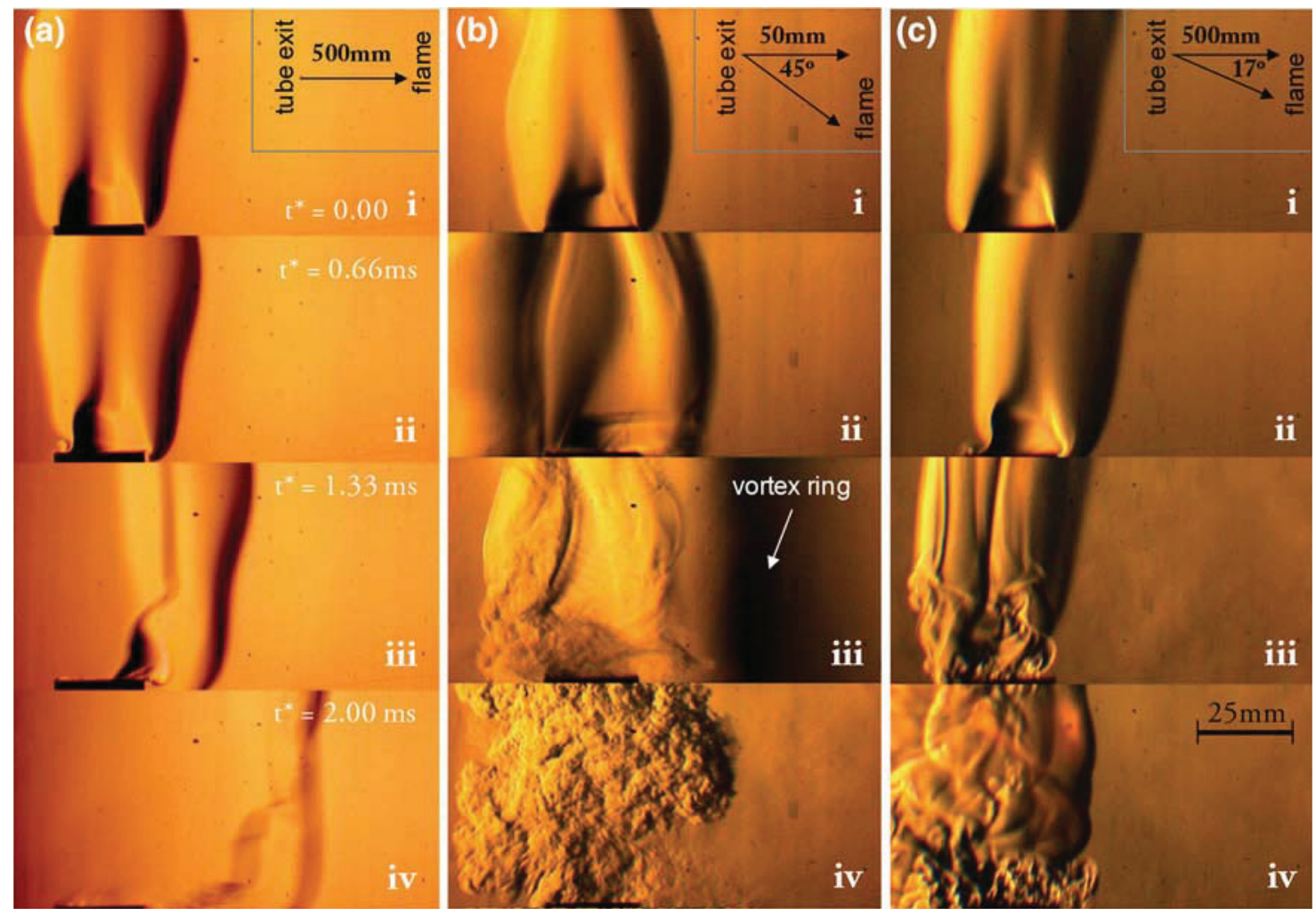

Fig. 2 Sequences from cases with flame positioned a on the shock tube axis $500 \mathrm{~mm}$ from the exhaust, b $50 \mathrm{~mm}$ from the exhaust at a $45^{\circ}$ angle, and c $500 \mathrm{~mm}$ from the exhaust at a $17^{\circ}$ angle

propagating downstream with the jet cause the flame to experience a highly rotational flow. This leads to a turbulent breakdown of the structure without significantly shifting the location of the flame itself. Such behaviour was consistent across all locations not in the direct line of the exhaust jet, though the strength and diameter of the vortex core and the more outward areas of recirculation were not directly measurable. The extensive swirling experienced by the flame appears to be enough to momentarily detach it from a steady fuel supply. Although significant heat remains in the immediate vicinity due to a lack of dissipative movement parallel to the exhaust flow, the combustion process is not able to restart due to the high local velocities in the vortex path. Only in Fig. 2b), with the flame close to the tube exit and offset at $45^{\circ}$, does flow close to the core of the vortex ring coincide with the schlieren's narrow plane of focus, and thus it is visualized in frame 2b) (ii) and (iii) passing through the flame directly-the exposure time for the frames does not facilitate a sharper depiction. In 2c), the vortex ring core is significantly off-image plane and weakened with distance, but the induced circulation in the flow remains significant as evidenced by the flame response.

Work now continues with a new, heavily instrumented shock tube with a combustive gas mixture recreating a pressure profile closer to that which could be produced with high explosives, and both largescale testing and numerical work will commence.

Acknowledgments This work was supported by a QANTAS Research Fellowship granted to Dr. Doig through the American Australian Association in 2011/2012, and UNSW internal funding. The authors are grateful to Mr. Duncan Chalmers of the UNSW School of Mining for use of his camera and supporting expertise.

\section{References}

Akhmetov DG (2001) Formation and basic parameters of vortex rings. J Appl Mech Tech Phys 42(5):794-805 Akhmetov DG (2009) Vortex rings. Springer, Heidelberg

Akhmetov DG, Lugovstov BA, Tarasov VF (1980) Extinguishing gas and oil well fires by means of vortex rings. Combust Explos Shock Waves 16(5):490-494 
Bliznetsov M, Dudin V, Gerasimov S, Houas L, Jourdan G, Logvinov A, Meshkov E, Vlasov Y (2001) Development of the method of interaction between shock wave and flame. In: Proceedings of the 8th international workshop on the physics of compressible turbulent mixing, Pasadena, USA, pp 1-5

Chandra N, Ganpule S, Kleinschmit NN, Feng R, Holmberg AD, Sundaramurthy A, Selvan V, Alai A (2012) Evolution of blast wave profiles in simulated air blasts: experiment and computational modeling. Shock Waves 22(5):403-415

Jiang Z, Wang C, Miura Y, Takayama K (2003) Three-dimensional propagation of the transmitted shock wave in a square cross-sectional chamber. Shock Waves 13(2):103-111. doi:10.1007/s00193-003-0197-y

Kashimura H, Yasunobu T, Nakayama H, Setoguchi T, Matsuo K (2000) Discharge of a shock wave from an open end of a tube. J Therm Sci 9(1):30-36. doi:10.1007/s11630-000-0042-x

Onodera O, Jiang ZL, Takayama K (1998) Holographic interferometric observation of shock waves discharged from an openend of a square cross-sectional shock tube. JSME Int J Ser B Fluids Therm Eng 41(2):408-415 